\title{
基于社交媒体数据的城市公园景感评价
}

\author{
郑天晨 ${ }^{1,2,3}$, 严 岩 ${ }^{1,2, *}$, 章 文 $^{1,4}$, 朱捷缘 ${ }^{1,2}$, 王辰星 ${ }^{1}$, 荣月静 ${ }^{1,2}$, 卢慧婷 ${ }^{1,2}$ \\ 1 中国科学院生态环境研究中心, 城市与区域生态国家重点实验室, 北京 100085 \\ 2 中国科学院大学, 北京 100049 \\ 3 比利时根特大学地理系, 根特 9000 \\ 4 武汉理工大学资源与环境工程学院, 武汉 430070
}

摘要:城市公园是城市居民与自然生态系统互动的主要场所,为居民提供重要的生态系统服务。从景感生态角度和方法对城市 公园文化服务进行评价,需要掌握大量居民个体对城市公园的感知、感受信息。随着现代信息技术和人们生活方式的发展,社 交媒体成为大众获取信息、发布观点的主要方式之一。社交媒体数据具有数据易于获取、时间跨度长、数据量大等优势,同时, 通过对社交媒体信息进一步进行文本提取与分析,还可以获取用户对特定活动场所更为具体的感知 和感受,因此,社交媒体数 据为城市公园景感评价提供了有价值的新的数据来源和手段。构建了基于社交媒体数据的城市公园景感评价框架和流程、评 价指标以及信息提取方法,包括对获取数据进行预处理; 构建感官感知及公园要素词库; 通过情感分类,形成积极和消极情感文 本, 表征用户对文本关联景感要素的满意度; 将各类情感文本分别进行要素及其对应感知感官的统计, 以频率衡量景感要素对 用户感知的重要性; 在此基础上,基于重要性-绩效分析 (IPA) 方法构建了感知评价模型。最后,以北京玉渊潭公园为例,进行 了实证研究。结果表明:视觉在居民对城市公园的感知体验中占据主导地位,触觉感知次之,听觉感知普遍不被重视,而嗅觉感 知评价相对缺失; 植物、周边人群、景观可识别性以及水体的视觉感知是最重要的景感要素; 基于感知评价模型,玉渊潭公园不 存在亟待改进的方面,次要改进的景感要素包括人群干扰、路面接触、食品售卖、感知风、观赏动物、人声、植物声、观赏道路 景观。

关键词:景感; 生态系统服务; 社交媒体; 城市公园; 感知

\section{Landsense assessment on urban parks using social media data}

ZHENG Tianchen $^{1,2,3}$, YAN Yan ${ }^{1,2, *}$, ZHANG Wen ${ }^{1,4}$, ZHU Jieyuan ${ }^{1,2}$, WANG Chenxing ${ }^{1}$, RONG Yuejing ${ }^{1,2}$, LU Huiting ${ }^{1,2}$

1 State Key Laboratory of Urban and Regional Ecology, Research Center for Eco-Environmental Sciences, Chinese Academy of Sciences, Beijing 100085, China 2 University of Chinese Academy of Sciences, Beijing 100049, China

3 Ghent University, Department of Geography, Ghent 9000, Belgium

4 Wuhan University of Technology, School of Resource and environmental engineering, Wuhan 430070, China

\begin{abstract}
The urban park is the place where dwellers interact with nature, contributing to urban sustainability by providing cultural ecosystem services. To evaluate the cultural ecosystem services of an urban park from the perspective of landsenses ecology, numerous information on visitors' perceptions and experiences is required. With the development of modern information technology as well as dwellers' lifestyles, the social media platform becomes one of the main places for the public to gain information and show opinions. Social media data is characterized by easy to obtain, long span of time, and a large quantity for landsense assessment of an urban park. By extracting and analyzing it, users' specific perceptions and
\end{abstract}

基金项目: 国家自然科学基金面上项目(71673268)

收稿日期: 2020-04-21; 网络出版日期:2021-09- 10

* 通讯作者 Corresponding author.E-mail: yyan@ rcees.ac.cn 
experiences of the given place can be obtained. This study established a framework of landsense assessment of an urban park using social media data and proposing a method for information extraction and indicator design. First, we preprocessed the data, and developed the corpus of senses and park elements, respectively. Then, the texts of positive and negative were divided by sentiment classification to measure users' satisfaction on the relative landsense elements. Based on the above, the perception assessment model was established using the Importance-Performance Analysis ( IPA) method. Finally, we conducted a landsense assessment of Yuyuantan Park in Beijing and suggested ways to improve visitors' perception. The conclusions are as follows: vision is a strongly dominant component in people's perception, following touch demission, while perceptions on the hearing dimension are relatively unimportant, and the indicator on smell dimension is still limited. 'Vision of plants', 'vision of humans', 'visual identification', 'vision of water' are the most important landsense elements. Through the perception assessment model, we did not identify the indicator that should be improved urgently, and the indicators that need to be improved secondly include 'vision of humans', ' touch of roads', 'food available' , 'feel of wind', 'vision of animals', 'sound of voice', 'sound of plants', and 'vision of roads'.

Key Words: landsense; ecosystem services; social media; urban park ; perception

城市公园作为城市居民与自然生态系统互动的场所, 为城市居民提供了户外休闲与活动的空间 ${ }^{[1-2]}$, 并 提供着重要的生态系统文化服务。生态系统文化服务 (CES) 指人类通过精神满足、认知能力的发展、思考、娱 乐和审美体验而从生态系统中获取的非物质收益 ${ }^{[3]}$ 。城市公园的文化服务可以提升城市居民的身体素质， 如增强骨骼、肌肉与心肺功能 ${ }^{[4]}$, 降低疾病风险 ${ }^{[5-6]}$, 也促进了城市居民的心理健康, 如减少压力 ${ }^{[7]}$, 缓解焦 虑 $^{[8]}$, 促进形成良好社会关系 ${ }^{[9-10]}$, 居民对文化服务的需求日益增加 ${ }^{[11]}$ 。

城市公园的文化服务的价值实现在于城市居民对文化服务的感知,因此,基于景感生态原理对城市公园 的文化服务进行评价与改进则尤为重要。景感生态理论 ${ }^{[12-13]}$ 以可持续发展为目标, 基于生态学的基本原理, 从自然要素、物理感知、心理感知、社会经济、过程与风险等相关方面, 研究土地利用规划、建设与管理的科学。 从个体感知的角度而言, 景感生态原理强调在规划、管理与决策中对人类所处的自然环境要素以及人类的物 理感知、心理感知等方面进行考虑 ${ }^{[14]}$ 。通过城市公园景感评价, 可探究公园现状对居民个体的影响, 充分考 虑在公园休闲的居民个体的感知感受,进一步满足居民的需求 ${ }^{[15]}$,并提升居民的景感满意度。

随着现代信息技术和人们生活方式的发展,尤其是互联网技术的普及,社交媒体作为大众获取信息、发布 观点的平台,存在着大量由用户参与的,对人物、事件、产品等有价值的评论信息 ${ }^{[16]}$, 是评估个人情绪、感知、 观点和兴趣的广泛数据来源 ${ }^{[17]}$ 。社交媒体数据易于获取、时间跨度长、数据量大 ${ }^{[18]}$, 体现了用户对特定活动 场所的具体感知和感受。因此, 基于社交媒体数据对城市公园进行景感评价, 可以掌握大量居民个体对城市 公园的感知、感受信息, 进而探究影响居民休闲体验的关键要素, 有助于推进景感的营造, 从而达到保持、改善 和增加生态系统服务的目的, 将人与生态系统服务紧密联系起来 ${ }^{[19]}$ 。

本文构建了基于社交媒体数据进行城市公园景感评价的框架, 通过生成感知与要素词库, 识别用户发布 的评论中存在的感官感知及其关联要素, 进而基于情感分析判断用户对所访问公园的满意度, 并以玉渊潭公 园为例,进行了实证评价。

\section{1 数据与方法}

\section{1 城市公园景感评价框架与流程}

城市公园的景感评价包括景感要素的重要性评价及满意度评价。本文构建了基于社交媒体数据进行城 市公园的景感评价的框架与流程(图 1)。通过识别指定公园的高频特征词,对景感评价指标体系进行调整; 通 过生成感知感官与公园要素词库, 对文本数据中各项景感要素指标进行识别与统计, 作为景感重要性评价结果; 对文本进行情感分类, 结合各景感要素指标的出现频率, 以消极评论比例作为各景感要素的满意度评价结果。 


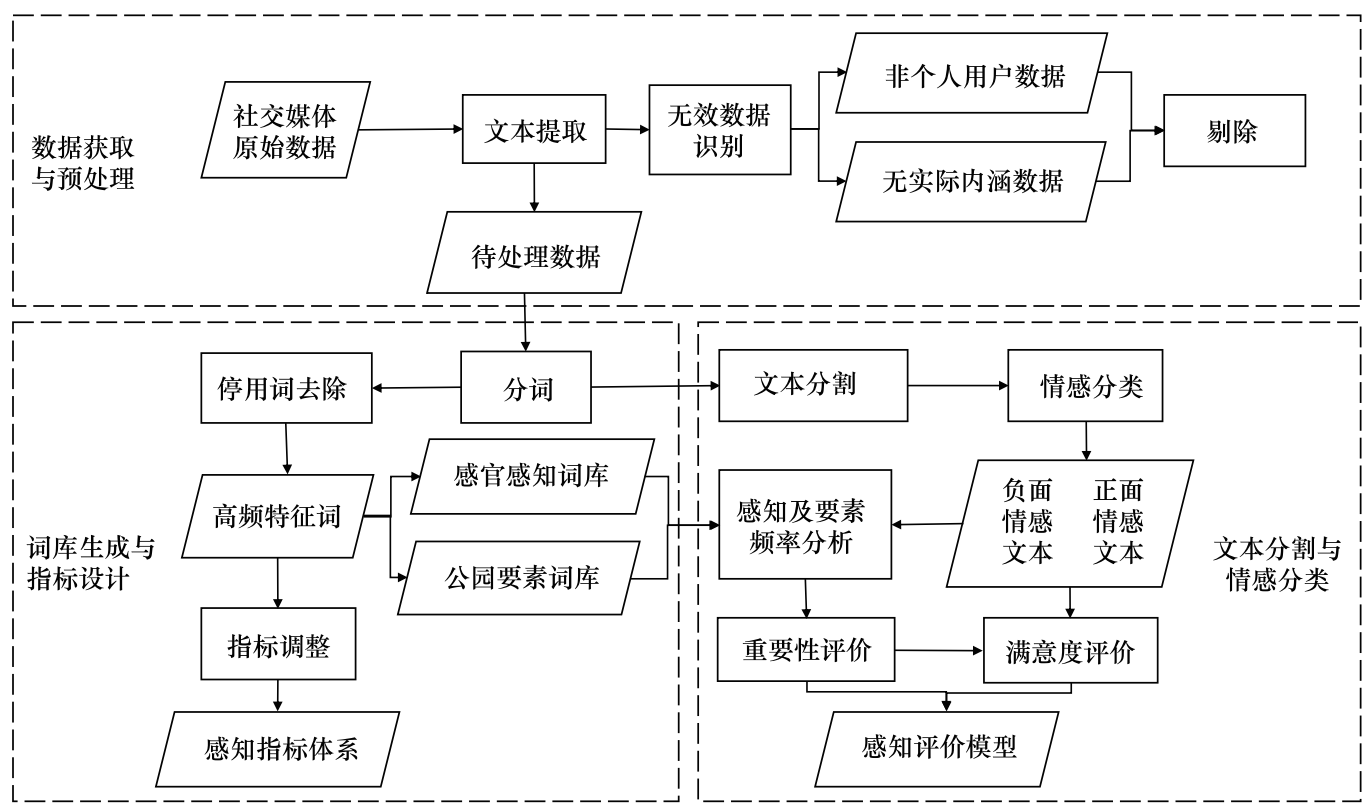

图 1 基于社交媒体数据的城市公园景感评价框架

Fig.1 Framework of landsense assessment of urban park based on social media data

\section{2 研究区概况}

本文选择北京市综合性公园之一玉沸潭公园作为研究区。玉渊潭公园位于北京市海淀区,占地面积 $132.38 \mathrm{hm}^{2}$, 其中水体面积为 $59.72 \mathrm{hm}^{2}$ 。园内分布有多个景点,包括樱花区、湿地区、运动休闲区和文化展示区 等四个主要功能区, 并设置有完善的基础设施, 可以购买多种食品及饮料。公园特色景点㭥花园, 是国内最大 的樱花专类园之一。除樱花外, 公园内配置有多样化的植物, 以水杉、柳为主的落叶树种和雪松为主的常绿树 种作为骨干植物, 少量种植连翘等花灌木, 部分道路两侧栽植有一、二年生草本花卉三色堇等。通过植物围合 出了多个小型活动空间,每个空间设计有不同的路面材质,包括水泥、石材、木质等。园内比较开阔的空间多 集中在水边, 水域内养殖了野鸭等动物, 但其他区域动物比较稀少, 很少听到鸟鸣等动物的声音。此外, 玉渊 潭公园在举办桜花文化活动、鲁冰花展等特色活动时,访问量极高,尤其在热门景点会聚集大量人群。

\section{3 数据与处理}

\subsection{1 数据的获取与预处理}

首先, 设定 “关键词” 为样本公园的名称, 提取社交媒体中与研究对象相关的信息。本文以用户发布的微 博评论作为数据来源, 以“玉渊潭”为关键词, 利用爬虫工具对 2018 年 9 月 30 日至 2019 年 9 月 29 日的相关 微博数据进行爬取, 获取数据 17975 条。

自动爬取的社交媒体数据通常存在大量噪声数据, 因此, 需对文本数据进行噪声数据的剔除。在本文中, 首先根据评论发布账户信息剔除了由公共账户发布的新闻信息、广告宣传信息及说明类文字,仅保留由个人 用户发布的原创内容。用户发布的评论中, 部分评论以图片内容为主, 往往文字描述过于简短(如仅包含公 园名称、所在地点) ; 同时,也存在内容虽提及了玉洲潭或玉渊潭公园、但实际描述内容或评价对象与公园关 联不大的评论。以上数据均视为无效数据, 需要进行剔除。因此, 本文在保留文本中随机抽取 20 条文本数据 进行人工判定其是否为有效数据, 并进行无效数据的剔除, 若存在无效数据, 则重复以上操作, 直至随机抽取 的数据均为有效文本数据。最终获得有效文本数据 12612 条。

1.3.2 词库生成与指标设计

相比英文词语之间由空格间隔,汉语书面语中的汉字逐个排列, 词语之间没有明显间隔,若要对其进行信 
息处理,应对等距排列的汉字串序列进行词的切分 ${ }^{[20]}$ 。因此,需使用分词工具对获取的文本数据进行分词处 理, 将原始连续文本拆分为词序列, 并过滤其中无实际含义的词语, 即停用词。本文选择采用结巴分词工具, 对微博文本数据进行了分词处理并去除停用词。随后,对文本数据进行词频分析,提取文本中的高频特征词 汇并生成词汇频率表。为避免歧义,本文在高频特征词的统计中,未统计单字出现的频率。

进而依据感知指标体系 ${ }^{[14]}$, 围绕个体的感官感知与公园的设计要素两个方面, 对高频特征词进行类别判 定, 分别形成感官感知与公园要素词库。其中, 感官感知包括视觉、听觉、嗅觉、触觉等类别, 公园要素包括人、 植物、水体、动物、道路、阳光、风、设施及景观可识别性等类别。本文将出现频率高于 10 的特征词进行了篎选 并逐条阅读,判断其是否属于感知感官及公园要素中的各类别范畴,并按照类别进行标签标注, 围绕各标签生 成感官感知与公园要素词库。同时, 基于玉渊潭公园的性质与特点, 对指标体系进行了调整, 增加了味觉感官 感知类别, 剔除了公园中不存在的水声、接触水等指标, 并将难以通过词库识别的空间感、噪声、异味等指标剔 除,形成了基于社交媒体数据的玉滟潭公园景感评价指标(表 1)。

表 1 玉渊潭公园景感评价指标

Table 1 Landsense indicator system of Yuyuantan Park, Beijing

\begin{tabular}{|c|c|c|}
\hline $\begin{array}{l}\text { 感官感知 } \\
\text { Senses }\end{array}$ & $\begin{array}{l}\text { 指标 } \\
\text { Indicators }\end{array}$ & $\begin{array}{l}\text { 指标内容 } \\
\text { Indicator definition }\end{array}$ \\
\hline \multirow[t]{6}{*}{ 视觉 Vision } & 人群干扰 & 人的数量适中、无干扰 \\
\hline & 观赏植物 & 观赏树、草、花等植物 \\
\hline & 观赏水景 & 观赏水景 \\
\hline & 观赏动物 & 观赏动物 (如野鸭) \\
\hline & 观赏道路景观 & 观赏道路的线形、色彩等 \\
\hline & 景观可识别性 & 观赏特别的景观 \\
\hline \multirow[t]{4}{*}{ 听觉 Hearing } & 人声 & 适度的人声 \\
\hline & 植物声 & 听风吹植物的声音 \\
\hline & 动物声 & 听动物的声音 (鸟鸣、虫鸣等) \\
\hline & 播报声 & 有广播播报 \\
\hline 嗅觉 Smell & 植物气息 & 闻到植物的气息 \\
\hline \multirow[t]{4}{*}{ 触觉 Touch } & 感知阳光 & 感受光影平衡 \\
\hline & 感知风 & 感受风环境 \\
\hline & 动物接触 & 没有蚊虫叮咬 \\
\hline & 路面接触 & 道路触感舒适 \\
\hline 味觉 Taste & 食品售卖 & 可在园中购买食品 \\
\hline
\end{tabular}

\subsection{3 文本分割与情感分类}

社交媒体数据文本中的每条内容往往含有多个维度的感官感知,体现着对样本公园的综合感知情况。因 此,需要通过对每条文本数据进行段落分割, 最大限度保证每条文本数据中的公园设计要素与感知感官的对 应。本文按 $5 \%$ 的比例对采集的社交媒体样本数据进行了人工信息识别,结果显示, $75.28 \%$ 的用户发表的评 论涉及景感要素比较单一,仅包含一种类型的景感要素;少部分用户发表了较长篇幅的评论,对自己的游园经 历进行具体描述,评论内容包括了多种景感要素。在这些评论中, $61.54 \%$ 的文本注重标点符号的使用, 因此, 本文采取通过“。” “? “!” “...”等标点符号进行文本分割的方式, 以保证大部分要素与感知感官的对应。

然后, 基于感官与要素词库, 对分割后的文本数据进行感知感官及其对应要素的判定。具体方法为:依据 感官感知词库, 首先分别笁选文本中存在视觉、听觉、嗅觉、触觉、味觉等感官感知词汇的文本, 进而根据公园 要素词库, 对各类别文本中是否含有相关词汇进行判断。最终获取 8178 条存在感知感官及对应要素的数据 作为有效样本数据。

文本情感分析又称意见挖掘, 是对带有情感色彩的主观性文本进行分析、处理、归纳和推理的过程 ${ }^{[16]}$, 可 
用于识别公众情绪、预测产品销售、衡量客户满意度等 ${ }^{[21]}$ 。其中, 情感极性分类是情感分析的基本任务 ${ }^{[22]}$, 旨在基于用户发布的书面文本,挖掘用户对特定产品或服务的观点,并进行文本情感倾向的划分 ${ }^{[23]}$ 。对公园 的景感满意度进行评价仅需获取用户对所访问公园的情感倾向,利用情感分析工具对有效文本数据进行情感 极性判断, 获取积极与消极两类情感判断结果。本文选择调用腾讯云自然语言处理 API 接口对文本数据进 行了情感极性分类。

\section{4 感知评价模型构建}

重要性-绩效分析 (IPA) 方法由 Martilla 和 James 提出,用于对比顾客消费前的期望和消费后的感知实绩 以及对各属性绩效表现状况的综合评价 ${ }^{[24-25]}$ 。自 20 世纪 90 年代初, IPA 分析法开始广泛应用于服务性行 业 $^{[26]}$,包括服务满意度评价 ${ }^{[27]}$ 、地区吸引力分析 ${ }^{[28]}$ 、旅游政策制定 ${ }^{[29]}$ 及景区满意度评价 ${ }^{[30]}$ 等。在满意度调 查中,IPA 方法要求受访者对指定调查对象的各项指标从重要性和满意度两个方面来进行评价, 形成 IPA 矩 阵。IPA 矩阵以游客期望程度 (重要性) 为横轴, 游客的满意程度 (表现性) 为纵轴, 并以总平均值作为 X-Y 轴 的分隔点, 将空间划分为四个象限, 各象限意义为:第一象限为继续努力发展区域; 第二象限为无需过多改善 区域;第三象限为缓慢改进区域;第四象限为需要重点改进区域。

利用 IPA 方法, 基于社交媒体文本分析结果, 进而对公园景感满意度进行评价。其中, 景感要素对用户 感知的重要性通过各要素出现频次进行衡量, 用户对各景感要素感知的满意度通过消极情绪文本在文本数据 中的占比表征。具体方法为基于情感分析结果笁选各感官感知及其关联要素, 并基于指标体系进行各指标对 应文本数量的频率统计; 同时, 针对各指标分别进行消极情感文本的数量统计, 以消极文本比例衡量用户对该 要素的不满意程度。

考虑到各景感要素之间等级的多层次划分 ${ }^{[14]}$, 在引人 IPA 方法的同时, 对指标的重要性和不满意度等级 均进行了重新划分, 分别以指标重要性、不满意度统计频率的下四分位数、上四分位数作为低-中、中-高等级 划分标准,据此形成呆待改进、次要改进、低优先级、继续保持、过度开发五个级别的感知评价模型(图 2)。基 于感知评价模型可确定公园改进策略的优先级别。

\section{2 结果}

由各类感官感知评论数量可知 (图 3), 用户对视觉感知的需求明显高于其他感官感知的需求, 对触觉感 知的需求相对偏高, 对听觉感知与味觉感知的需求相对偏少, 对嗅觉感知需求最小。在满意度方面, 用户在与 味觉感知和听觉感知等感官感知相关的评论中消极评论占比最高, 与触觉感知和嗅觉感知相关的评论中消极 评论占比偏高, 而对视觉感知的消极评论占比最小。

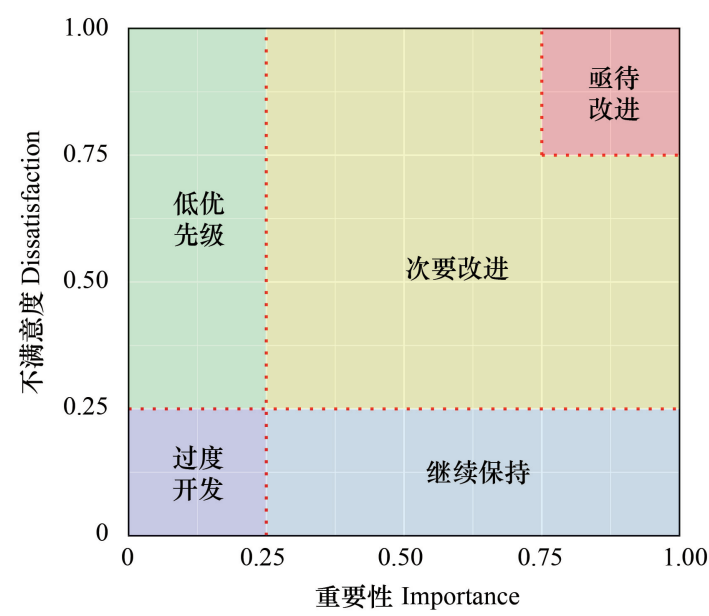

图 2 感知评价模型 [14]

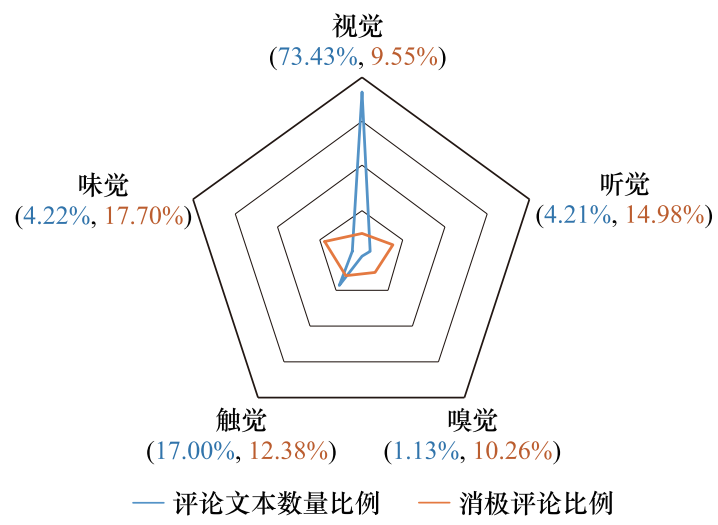

图 3 基于感官的景感重要性及满意度对比

Fig. 3 Comparison of landsense importance and satisfaction based on five senses

Fig.2 Perception assessment model 
由各景感要素感知评论数量可知 (表 2), 用户对观赏植物、人群干扰、景观可识别性和观赏水景的重视程 度明显高于其他景感要素, 而明显不重视的景感要素包括动物声、动物接触、播报声和植物气息。在用户的满 意度评价中, 人声、动物声、感知风和食品售卖等景感要素的消极评论比例偏高, 而景观可识别性、感知阳光、 观赏水景和观赏植物等景感要素的消极评论占比偏低。

表 2 各指标评论文本比例

Table 2 Percentage of comments and negative comments

\begin{tabular}{lcc}
\hline 指标 & $\begin{array}{c}\text { 评论文本数量比例/\% } \\
\text { Percentage of comments }\end{array}$ & $\begin{array}{c}\text { 消极评论比例 } / \% \\
\text { Percentage of negative comments }\end{array}$ \\
\hline 观赏植物 Vision of plants & 34.61 & 8.55 \\
人群干扰 Vision of humans & 15.63 & 14.46 \\
景观可识别性 Visual identification & 9.35 & 6.43 \\
观赏水景 Vision of water & 7.26 & 8.16 \\
路面接触 Touch of roads & 6.95 & 12.97 \\
感知阳光 Feel of sunlight & 5.32 & 7.48 \\
食品售卖 Food available & 4.22 & 17.70 \\
感知风 Feel of wind & 4.10 & 17.73 \\
观赏动物 Vision of animals & 3.76 & 9.28 \\
观赏道路景观 Vision of roads & 2.83 & 8.90 \\
植物声 Sound of plants & 1.92 & 12.63 \\
人声 Sound of voice & 1.29 & 20.30 \\
植物气息 Smell of plants & 1.13 & 10.26 \\
播报声 Sound of broadcast & 0.90 & 11.83 \\
动物接触 Contact with animals & 0.63 & 12.31 \\
动物声 Sound of animals & 0.10 & 20.00 \\
\hline
\end{tabular}

由感知评价模型可知 (图 4), 不存在函待改进的景 感要素,次要改进的景感要素为人群干扰、路面接触、食 品售卖、感知风、观赏动物、人声、植物声、观赏道路景 观, 可以继续保持的景感要素包括景观可识别性、观赏 植物、感知阳光、观赏水景; 而过度开发的要素包括动物 声、动物接触、播报声和植物气息。

\section{3 讨论}

\section{1 玉渊潭公园景感评价结果分析}

北京玉渊潭公园的景感评价案例研究表明,视觉在 城市公园的感知体验中依然占据主导地位, 触觉感知次 之, 而居民普遍不重视听觉感知, 这与先前的研究结果 一致 ${ }^{[14]}$ 。而嗅觉在距离型感知器官中可发挥作用的尺 度最有限, 导致了嗅觉感知指标相对缺失, 而在社交媒 体数据中嗅觉感知的体现同样偏少, 可见感官的发挥作 用的尺度会显著影响其感知情况。作为北京市综合性 公园之一,玉渊潭公园为城市居民提供了食品、饮料售 卖等服务, 本文仅设置了“食品售卖”一项味觉感知指 标, 由于指标数量有限, 结果显示居民对味觉感知体验

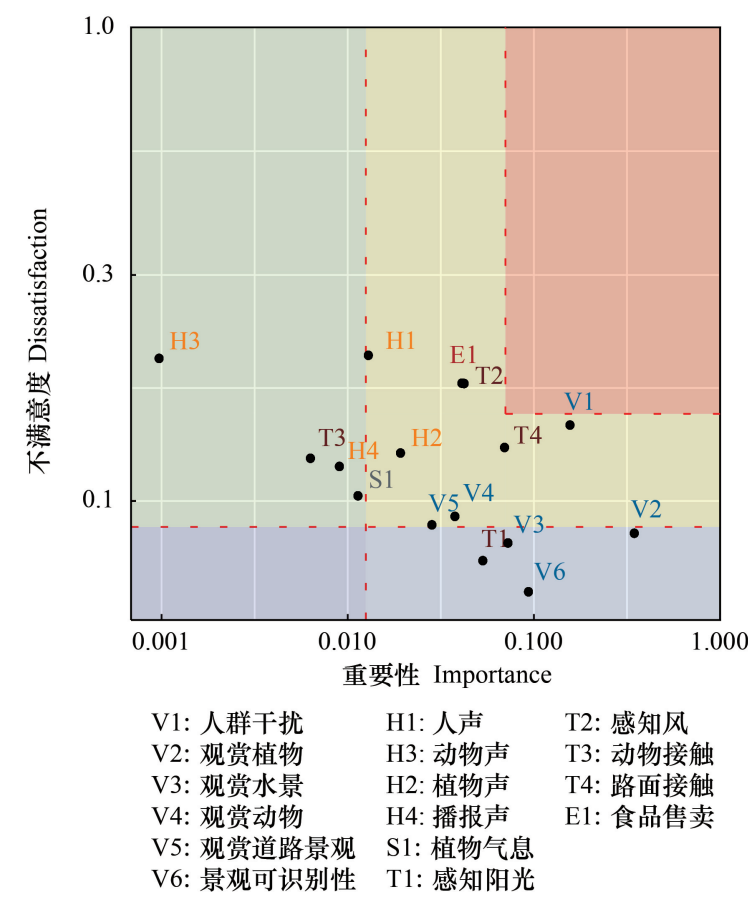

图 4 北京玉渊潭公园感知评价模型

Fig.4 Perception assessment model of Yuyuantan Park, Beijing 
的重视程度相对偏低。

对景感要素而言, 在玉沸潭公园内,植物、周边人群、景观可识别性和水体的视觉感知是最重要的景感要 素之一, 显示出城市居民对亲近自然、远离人群干扰的需求以及对公园特色的关注。与先前结果有较大差异 的是, 基于社会调查的结果表明居民对动物声音比较重视 ${ }^{[14]}$, 然而, 在社交媒体数据中, 用户很少提及对动物 声音的感知,这可能与用户在玉洲潭公园中感知动物声音比较困难有关。

基于研究结果, 主要围绕访问者的视觉、听觉感知,道路的视觉、触觉感知等方面,对玉渊潭公园提出以下 设计与管理建议, 以促进景感营造:

访问者: 通过完善公园景观与设施布局,适量增设游唕设施, 提高公园游客承载量; 加强公园管理技术与 手段, 运用物联网技术, 建立景感满意度生态风险管理平台 ${ }^{[14,31-32]}$, 对公园人群密度进行实时监测, 引导居民 合理规划游园路线。

动植物: 合理配置园区植物, 适当增加常绿灌丛的栽植种类与密度, 为居民提供避风、隔音的小尺度休闲 空间; 保证园内小型野生动物栖息地面积,为居民提供更多观赏动物的机会。

人工设施: 依据居民需求增设公园零售商品及游乐设施种类,并合理设定价格; 丰富主干道路景观,并结 合指示牌引导居民选择合适的游园路径。

3.2 社交媒体数据在城市公园景感评价中的应用前景

相比传统社会调查, 基于社交媒体数据对城市公园文化服务进行景感评价, 耗时短, 可获得大量样本数 据, 并且往往体现着用户对城市公园的综合感知情况, 可获取用户对特定活动场所更为具体的感知和感受, 并 识别最影响居民体验的景感要素。同时,社交媒体数据的综合性也体现了对心理感知识别的可能性, 后续研 究可围绕社交媒体数据中用户的评论, 对多种感知进一步识别与区分, 构建包括物理感知、心理感知等方面的 综合性感知评价指标体系。

然而, 由于中文文本的特征, 在利用社交媒体数据进行城市公园文化服务的感知评价时, 依然存在需要进 行优化的方向。首先, 噪声数据的剔除依然比较困难。新闻文本、广告等非个人用户发布的信息的剔除难以 通过机器学习的方法进行识别, 依然需要人工判断, 这在利用大量数据文本进行景感评价时是难以实现的。 其次, 尽管社交媒体数据量非常大, 可用于景感评价的样本依然十分有限, 这表明基于感官感知的识别依然比 较困难,需要对感知与要素词库进一步扩充,并基于大数据分析方法, 对感官感知及其对应要素进行识别训 练。此外, 部分评论文本篇幅较长, 内容复杂, 含有较多的感官感知与公园要素词汇, 需要进一步改进基于词 库进行景感要素的识别方法,保证感知与要素的对应关系。

\section{4 结论}

基于景感生态原理对生态系统文化服务进行评价, 是扩展文化服务研究深度的新思路。本文构建了基于 社交媒体数据的城市公园景感评价框架和流程、评价指标以及信息提取方法,并以北京玉渊潭公园为例进行 了实证评价。结果表明, 城市居民更关注在城市公园中的视觉感知体验, 触觉感知次之, 而居民普遍不重视听 觉感知, 这与先前通过社会调查方法得到的研究结果一致; 其中, 植物、周边人群、景观可识别性以及水体的视 觉体验受到了大多数居民的重视; 最后基于感知评价模型提出了玉渊潭公园的设计与管理改进建议。基于社 交媒体数据进行城市公园景感评价的优势在于数据获取耗时短、数据量大, 并且可以获取用户对特定活动场 所更具体、更直接的感知和感受, 有助于更具有针对性的改进建议的提出; 此外, 社交媒体数据的综合性为识 别居民访问公园时的心理感知提供了可能性, 为基于景感生态理论的城市公园综合感知评价提供了数据基 础。然而, 相比社会调查方法, 社交媒体数据在城市公园文化服务的景感评价中依然具有局限性。首先, 噪声 数据的剔除主要依赖于人工识别, 当数据量过大时往往难以实现; 其次, 景感要素指标的识别需要更有效的识 别方法与覆盖范围更广泛的词库。但是, 随着信息技术的发展与互联网用户的不断增加, 通过社交媒体获取 的数据与社会调查数据之间的差异逐步缩小, 基于大数据对城市公园进行景感评价将具有更高的可操作性, 
是景感生态理论与文化服务评价的发展方向之一。

\section{参考文献( References) :}

[ 1 ] Hayward D G, Weitzer W H. The public's image of urban parks: past amenity, present ambivalance, uncertain future. Urban Ecology, 1984, 8 (3) : 243-268.

[ 2 ] Jim C Y, Chen W Y. Recreation-amenity use and contingent valuation of urban greenspaces in Guangzhou, China. Landscape and Urban Planning, 2006, 75(1/2): 81-96.

[ 3 ] Millenium Ecosystem Assessment. Ecosystems and Human Well-Being: Synthesis. Washington: Island Press, 2005.

[ 4 ] Lee A C K, Maheswaran R. The health benefits of urban green spaces: a review of the evidence. Journal of Public Health, 2011, 33(2) : $212-222$.

[ 5 ] Mitchell R, Popham F. Effect of exposure to natural environment on health inequalities: an observational population study. The Lancet, 2008, 372 (9650): 1655-1660.

[ 6 ] Samawi H M. Daily walking and life expectancy of elderly people in the Iowa 65+ rural health study. Frontiers in Public Health, 2013 , $1: 11$.

[ 7 ] Ulrich R S. Natural versus urban scenes: some psychophysiological effects. Environment and Behavior, 1981, 13(5): 523-556.

[ 8 ] Kaplan S. Meditation, restoration, and the management of mental fatigue. Environment and Behavior, 2001, 33(4) : 480-506.

[ 9 ] Coley R L, Sullivan W C, Kuo F E. Where does community grow?: the social context created by nature in urban public housing. Environment and Behavior, 1997, 29(4): 468-494.

[10] Kuo F E, Bacaicoa M, Sullivan W C. Transforming inner-city landscapes: trees, sense of safety, and preference. Environment and Behavior, 1998, 30(1): 28-59.

[11] Guo Z W, Zhang L, Li Y M. Increased dependence of humans on ecosystem services and biodiversity. PLoS One, 2010, 5(10): e13113.

[12] Zhao J Z, Liu X, Dong R C, Shao G F. Landsenses ecology and ecological planning toward sustainable development. International Journal of Sustainable Development \& World Ecology, 2016, 23(4) : 293-297.

[13] Dong R C, Liu X, Liu M L, Feng Q Y, Su X D, Wu G. Landsenses ecological planning for the Xianghe Segment of China's Grand Canal. International Journal of Sustainable Development \& World Ecology, 2016, 23(4) : 298-304.

[14] Zheng T C, Yan Y, Lu H T, Pan Q, Zhu J Y, Wang C X, Zhang W, Rong Y J, Zhan Y J. Visitors' perception based on five physical senses on ecosystem services of urban parks from the perspective of landsenses ecology. International Journal of Sustainable Development \& World Ecology, $2020,27(3): 214-223$.

[15] 严岩, 朱捷缘, 吴钢, 詹云军. 生态系统服务需求、供给和消费研究进展. 生态学报, 2017, 37 (8) : 2489-2496.

[16] 赵妍妍, 秦兵, 刘挺. 文本情感分析. 软件学报, 2010, 21(8): 1834-1848.

[17] Do Y. Valuating aesthetic benefits of cultural ecosystem services using conservation culturomics. Ecosystem Services, 2019, $36: 100894$.

［18］周胜臣, 㫿文婷, 石英子, 施询之, 孙韵辰. 中文微博情感分析研究综述. 计算机应用与软件, 2013, 30(3) : 161-164, $181-181$.

[19] Zhao J Z, Yan Y, Deng H B, Liu G H, Dai L M, Tang L N. Remarks about landsenses ecology and ecosystem services. International Journal of Sustainable Development \& World Ecology, 2020, 27(3): 196-201.

[20］刘迁, 贾惠波. 中文信息处理中自动分词技术的研究与展望. 计算机工程与应用, 2006, 42(3): 175-177, 182- 182.

[21] Ravi K, Ravi V. A survey on opinion mining and sentiment analysis: tasks, approaches and applications. Knowledge-Based Systems, 2015, 89: 14-46.

[22] Cambria E, Schuller B, Xia Y Q, Havasi C. New avenues in opinion mining and sentiment analysis. IEEE Intelligent Systems, 2013, 28(2): 15-21.

[23] Ye Q, Zhang Z Q, Law R. Sentiment classification of online reviews to travel destinations by supervised machine learning approaches. Expert Systems with Applications, 2009, 36(3): 6527-6535.

[24] Martilla J A, James J C. Importance-performance analysis. Journal of Marketing, 1977, 41(1) : 77-79.

[25] 梁慧, 王远坤, 刘明霞. 游客对旅游目的地地方美食体验感知的 IPA 分析一以湖北恩施州为例. 农林经济管理学报, 2016, 15(3): 335-342.

[26] 陈旭. IPA 分析法的修正及其在游客满意度研究的应用. 旅游学刊, 2013，28(11)：59-66.

[27] Chen P Z, Liu W Y. Assessing management performance of the national forest park using impact range-performance analysis and impact-asymmetry analysis. Forest Policy and Economics, 2019, 104: 121-138.

[28] Go F, Zhang W. Applying importance-performance analysis to Beijing as an international meeting destination. Journal of Travel Research, 1997, 35 (4) : 42-49.

[29] Evans M R, Chon K S. Formulating and evaluating tou rism policy using importance-performance analysis. Hospitality Education and Research Journal, 1989, 13(3): 203-213.

[30] Sever I. Importance-performance analysis: a valid management tool?. Tourism Management, 2015, 48: 43-53.

[31] Tang L N, Wang L, Li Q Y, Zhao J Z. A framework designation for the assessment of urban ecological risks. International Journal of Sustainable Development \& World Ecology, 2018, 25(5): 387-395.

[32] Wang H W, Zhu L C, Zhao C Y, Zheng S N. Urban ecological risk assessment management platform. International Journal of Sustainable Development \& World Ecology, 2018, 25(5): 477-482. 